\title{
How Useful are Weather Satellites?
}

THE recent publication of a technical note on "The Use of Satellite Pictures in Weather Analysis and Forecasting" is an opportune moment to ask how useful weather satellites are proving to be. The purpose of the note, published by the World Meteorological Organization, is to help with the interpretation of pictures of cloud formations.

TIROS 1, launched in 1960, was the first satellite designed specifically for meteorological observations. It was followed by a series of TIROS and NIMBUS satellites which tested different camera systems and developed the techniques needed for an operational weather watching system. Once the development work was completed, the running of the project in the United States was transferred from the National Aeronautics and Space Administration to the Environmental Science Services Administration. The first satellite in the operational system was ESSA 1, launched early in 1966.

Weather satellites are used extensively in Britain as an aid in the plotting of weather charts. At present between 20 and 25 pictures a day are received by the Meteorological Office at Bracknell from NIMBUS 2one of the research and development satellites still functioning - and from ESSA 6. The ESSA satellite has an automatic picture transmission facility, which means that any station in the line of sight can in principle receive pictures of conditions in the area. The forecasters at Bracknell thus have available pictures of cloud formations over large areas of the North Atlantic and Europe, sometimes only 20 minutes after they were taken.

The satellite pictures are used to correct the surface charts-the familiar maps showing the positions of fronts and isobars delineating regions of high and low pressure. The procedure is to compare the cloud distribution implied by the surface chart with that revealed by the satellite pictures. Where the two differ, a correction is made to the surface chart. Pictures of the cloud cover can also be taken during the night with infrared sensors. Such an instrument is fitted to ESSA 6 but has not worked for some time.

It is clear that weather satellites do not eliminate the need for ground observing stations. This is particularly so because there seems to be no immediate prospect of developing satellite-borne instruments for measuring pressures and wind speeds in the lower atmosphere - two parameters of great importance to forecasters. As far as meteorologists are concerned, the full benefits of earth satellites have yet to be realized. Before any really revolutionary improvements in weather forecasting are made, there will have to be many more weather stations, particularly in remote polar and oceanic areas and in the underdeveloped nations. These regions still account for enormous areas of uncertainty on the surface charts.
In addition to stations on land and fixed to buoys at sea, there are plans for the introduction of free-floating balloons to carry instruments over remote regions. Experiments with balloons of long lifetime have been made by the French space agency and by the National Centre for Atmospheric Research, Colorado. The Americans have released a series of 'Mylar' plastic balloons from New Zealand and from McMurdo Sound in the Antarctic. The balloons float at a height where. the atmospheric pressure is equal to the pressure inside the balloon. Most of the American balloons were inflated so as to drift at levels between 18,000 feet and 40,000 feet. Some of them have lasted for more than two hundred days, circumnavigating the earth several times until lost because of icing. To test the satellite data collection aspects of this project, the satellite NIMBUS B is to be launched in April. This will be able to locate and interrogate free-floating balloons and relay the information to ground stations. In the first instance tests will be made with a balloon drifting over the United States. It therefore seems probable that in the $1970 \mathrm{~s}$, communication satellites will be the chief contribution of spacs technology to meteorology.

\section{Cerebral Impasse}

from our Neurophysiology Correspondent

UNDER the title "The Logical Analysis of Cerebral Functions" a meeting was held at the Royal Society on February 8 under the organization of Professors J. Z. Young and H. C. Longuet-Higgins.

Professor Weiskrantz began his paper with a plea for illogicality in research. This at first seemed at variance with the spirit of the meeting, but he proceeded to give a logical analysis of the effects of temporal lobe lesions on the classification of visual information by monkeys and man. His thesis that engineering analogies often die while the real nervous system survives carries some weight, although a distinction should be made between a logical function and the device which performs it. It is fashionable to compare retrieval by the cerebral cortex with holography and information storage. This is a useful analogy because it presents in tangible terms a system capable of performing some of the more mysterious operations of the brain: it is not, of course, suggested that the brain is a hologram but merely that transformation of input into some kind of storable interference pattern is a possible mechanism of memory.

Professor Longuet-Higgins presented his theory of the temporal storage of patterns by "holophony" (Nature, 21\%, 104). He showed that a bank of resonators could store information about the component frequencies of an auditory input and then reproduce the whole input when stimulated again by only a fragment of it, provided that the coupling constant of each resonator were altered proportionally to the power of the input signal in its frequency band. After his presentation the 